\title{
Small-Signal Analysis of DC-DC Converters with Sliding Mode Control
}

\author{
Paolo Mattavelli, Member, IEEE, Leopoldo Rossetto, Member, IEEE, and Giorgio Spiazzi, Member, IEEE
}

\begin{abstract}
This paper deals with small-signal analysis of dc-dc converters with sliding mode control. A suitable small-signal model is developed which allows selection of control coefficients, analysis of parameter variation effects, characterization of the closed-loop behavior in terms of audiosusceptibility, output and input impedances, and reference to output transfer function. Unlike previous analyses, the model includes effects of the filters used to evaluate state variable errors. Simulated and experimental results demonstrate model potentialities.
\end{abstract}

Index Terms - Control techniques, sliding mode control, smallsignal analysis

\section{INTRODUCTION}

A CONTROL technique suitable for dc-dc converters must cope with their intrinsic nonlinearity and wide input voltage and load variations, ensuring stability in any operating condition while providing fast transient response. Among the various control techniques proposed in literature, sliding mode control [1], [2] offers several advantages, namely, largesignal stability, robustness, good dynamic response, and simple implementation [3], [4].

In spite of these positive aspects, sliding mode control is not yet popular, probably because its theoretical complexity which can make selection of controller parameters difficult. In fact, these parameters must be chosen so as to satisfy existence, hitting, and stability conditions. The analysis is easily carried out only for second-order converters which permit a phaseplane description of the system.

Another limitation is that sliding mode control requires, in theory, sensing of all state variables and generation of suitable references for each of them.

In practice, generation of reference signals for all state variables is not needed in $\mathrm{dc}-\mathrm{dc}$ converters. In fact, since only error signals are required for the control, they can be achieved by high-pass filtering the state variable signals in the assumption that their dc component is automatically adjusted by the converter according to the input-output power balance condition. Of course, these high-pass filters, not considered in previous analyses, increase the system order and can heavily affect the converter dynamic.

Moreover, converter control can be done effectively by sensing only one inductor current in addition to the output

Manuscript received August 2, 1995; revised June 25, 1996.

P. Mattavelli and L. Rossetto are with the Department of Electrical Engineering, 35131 Padova, Italy.

G. Spiazzi is with the Department of Electronics and Informatics University of Padova, 35131 Padova, Italy.

Publisher Item Identifier S 0885-8993(97)00412-2. voltage [4], even for high-order converters (e.g., Cuk and Sepic), resulting in a control complexity similar to that of standard current-mode controllers. However, a comparison between the two solutions (full-order control and reducedorder control) is not easy to obtain.

In summary, there is a lack of models able to describe the effects of circuit and controller parameter variations and to allow a comparison between the sliding mode approach and other popular control techniques like current-mode control, pulse width modulation (PWM) control, etc., in terms of converter transfer functions (audiosusceptibility, output and input impedances, and reference to output transfer ratio).

This paper presents a small-signal model of sliding modecontrolled $\mathrm{dc}-\mathrm{dc}$ converters operating in a continuous conduction mode which also includes the effects of the filters used to determine state variable references. Simulated results of a Sepic converter are reported which show the model potentialities. Experimental results of a Boost converter are also given.

\section{BASic Sliding Mode Controller}

Fig. 1 shows the general sliding mode-control scheme of $\mathrm{dc}-\mathrm{dc}$ converters. Although not used in practice, this scheme emphasizes the properties and operation mechanism of this control. $U_{g}$ and $u_{C N}$ are input and output voltages, respectively, while $i_{L i}$ and $u_{C j}(i=1 \div r, j=r+1 \div N-1)$ are the internal state variables of the converter ( $r$ inductor currents and $N-r-1$ capacitor voltages), and $N$ is the system order.

For the sake of generality, the state variables will be indicated as components of state vector $\widetilde{x}$. According to the general sliding mode-control theory, all state variables are sensed, and the corresponding errors (defined by difference to the reference values $\tilde{x}^{*}$ ) are multiplied by proper gains $K=\left[k_{1}, k_{2}, \cdots k_{N}\right]^{T}$ and added together to form sliding function $\psi$. Then, hysteretic block HC keeps this function near zero by gating the power switch $S$ on and off. We can therefore assume

$$
\psi=K^{T}\left(\tilde{x}-\tilde{x}^{*}\right) \cong 0
$$

This means that the control forces the system to evolve on the hyperplane (sliding surface) defined by (1).

As discussed in [4], selection of coefficients $K$ must be done in order to satisfy some basic requirements: 1) the state trajectories starting from points near the hyperplane must be directed toward the sliding surface (1) for both possible states of the converter switch (existence condition); 2) the system 


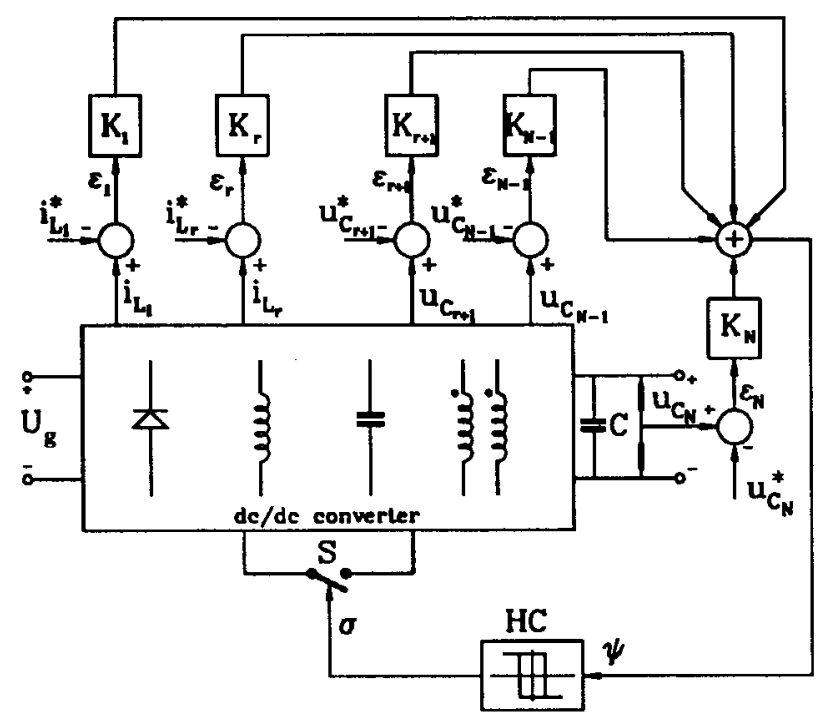

Fig. 1. Basic scheme of a sliding mode controller for dc-dc converters.

trajectories must encounter the sliding surface irrespective of their starting point in the phase space (hitting condition); and 3 ) the system motion on the sliding surface must reach a stable point corresponding to the desired voltages and currents (stability condition).

Although the feasibility of a sliding mode controller of reduced order (where only one inductor current is sensed in addition to the output voltage) has been already demonstrated [4], in the following we will consider the case of Fig. 1 for the sake of generality. Results for reduced-order controllers are easily obtainable by setting some sliding coefficients, $k_{i}$, to zero.

\section{SMAll-Signal Model Derivation}

A generic dc-dc converter, working in a continuous conduction mode, can be characterized by the following two sets of equations:

$$
\begin{array}{ll}
\dot{\tilde{x}}=A_{1} \tilde{x}+B_{1} \tilde{u} & \text { switch on } \\
\dot{\widetilde{x}}=A_{0} \tilde{x}+B_{0} \tilde{u} & \text { switch off }
\end{array}
$$

where $\widetilde{x}$ is the vector of state variables, and $\tilde{u}$ is the vector of the input variables (input voltage $\tilde{u}_{g}$ and possibly load current $\left.\tilde{i}_{o}\right)$. According to the state space averaging method [5], the average system behavior is described by the equation

$$
\dot{x}=A x+B u
$$

where

$$
\begin{aligned}
& A=A_{1} \delta+A_{0}(1-\delta) \\
& B=B_{1} \delta+B_{0}(1-\delta)
\end{aligned}
$$

where $\delta$ is the converter duty cycle in the steady state, and $x$ and $u$ are the averaged state variables and input variables, respectively. After linearization (small-signal assumption), we can derive the following small-signal equation in which the hat means perturbation from a steady-state working point $(X, U)$

$$
\dot{\hat{x}}=A \hat{x}+B \hat{u}+C \hat{\delta} .
$$

Matrices $A$ and $B$ are the same as (4b), and matrix $C$ is given by

$$
C=\left(A_{1}-A_{0}\right) X+\left(B_{1}-B_{0}\right) U
$$

In general, only some of the reference values $\tilde{x}^{*}$ indicated in (1) are fed from the external control (often, only as output voltage reference is assigned). The other references are derived internally to the controller, normally by low-pass filtering the corresponding state variables. For these latter references we can define $M$ additional state variables $x_{\text {int }}^{*}$ whose dynamic is described by

$$
\dot{x}_{i_{\mathrm{int}}}^{*}=-\frac{1}{\tau_{i}} x_{i_{\mathrm{nnt}}}^{*}+\frac{1}{\tau_{i}} x_{i} \quad i=1 \div M
$$

where $\tau_{i}$ are the corresponding filter time constants. Thus, the system order increases by $M$. The remaining $N-M$ state variable references are additional inputs. Note that linear (7) holds also for perturbed variables.

We call $\hat{x}^{\prime}$ the vector of all state variables, including the additional state variables of the filters

$$
\hat{x}^{\prime}=\left[\begin{array}{c}
\hat{x} \\
\hat{x}_{\mathrm{int}}^{*}
\end{array}\right] .
$$

Now we can exploit condition (1) which holds whenever the converter operates in the sliding mode. Since the hysteretic block HC forces the instantaneous state variables close to their references (the closeness depends on the hysteresis amplitude and thus on the switching frequency), constraint (1) can be used also for averaged variables. Thus, taking the derivative of (1), i.e., $\dot{\psi}=0$, and using (5) and (7), we can express the converter duty cycle as a function of complete state vector $\hat{x}^{\prime}$, derivative of external references $\dot{\hat{x}}_{\text {ext }}^{*}$, and input variables

$$
\hat{\delta}=D_{1} \hat{x}^{\prime}+D_{2} \hat{u}+D_{3} \dot{\hat{x}}_{\text {ext }}^{*}
$$

where matrices $D_{i}(i=1 \div 3)$ are given in Appendix I. Substituting (9) in (5) and using (7) we obtain the linear system equation

$$
\dot{\hat{x}}^{\prime}=A_{c} \hat{x}^{\prime}+B_{c} \hat{u}+D_{c} \dot{\hat{x}}_{\text {ext }}^{*}
$$

Taking now into account constraint (1), which holds even for perturbed variables, the system order is reduced by one. The final small-signal model has order $N+M-1$ and is given by

$$
\dot{\hat{x}}^{\prime \prime}=A_{T} \hat{x}^{\prime \prime}+B_{T} \hat{u}+D_{T 1} \hat{x}_{\mathrm{ext}}^{*}+D_{T 2} \dot{\hat{x}}_{\mathrm{ext}}^{*}
$$

Expressions for matrices $A_{c}, B_{c}, D_{c}, A_{T}, B_{T}, D_{T 1}$, and $D_{T 2}$ are given in Appendix I.

From (11) we are now able to compute all closed-loop transfer functions. In particular, the input-output voltage transfer function (audiosusceptibility), external reference to output voltage, and input admittance are directly derived from (11). 


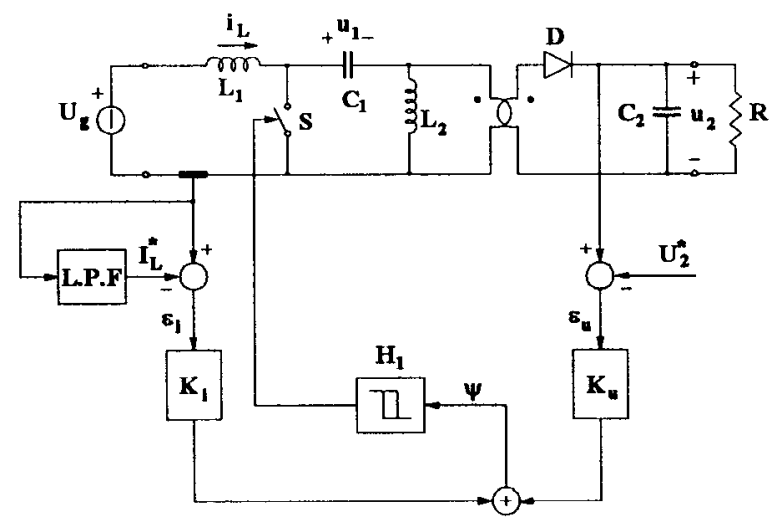

Fig. 2. Sepic converter with sliding mode control.

Instead, derivation of converter output impedance requires definition of the load current as an external input in (5).

As shown above, the model derivation involves only knowledge of the small-signal model derived from the state space averaging method and condition (1) which holds whenever the system moves on the sliding surface. Thus, a similar procedure can be applied for the derivation of the model for converters operating in discontinuous conduction mode (DCM) by simply using the corresponding small-signal model for the power stage [5] and thus changing (4b). However, the validity of the model is guaranteed only if a proper sliding surface is chosen so as to ensure a stable sliding mode motion for converters operating in DCM.

It is noticeable that the above model derivation involves the same approximations of state space-averaged modeling [5]. Thus, its validity is limited to those frequencies for which switching frequency effects can be neglected. In this view, the hysteresis block HC is modeled as constraint (1) on the average values without considering the effects due to the switching frequency.

\section{MODEL VERIFICATION}

In order to test the validity of the model, a Sepic converter (whose parameters are given in Appendix IV) with a sliding mode control was analyzed. According to [6], only two state variables are sensed: one is the output voltage, and the other is the input inductor current as shown in Fig. 2.

Output impedance, audiosusceptibility, input admittance, and reference to output transfer function were computed from the above small-signal model. The corresponding results are shown in Fig. 3. For the output impedance [Fig. 3(a)] and audiosusceptibility [Fig. 3(b)], the results of the model simulation (continuous line) are reported together with the results of the converter simulation (dotted line) for the purpose of comparison. As we can see, the maximum error is about $1 \mathrm{~dB}$ in the whole range of operating frequencies. Fig. 3(c) shows the converter input admittance, and Fig. 3(d) shows the reference to output transfer function obtained from our model.

As already explained, the proposed small-signal model can be used both for full and reduced-order control, thus showing how much the latter influences the system performances.

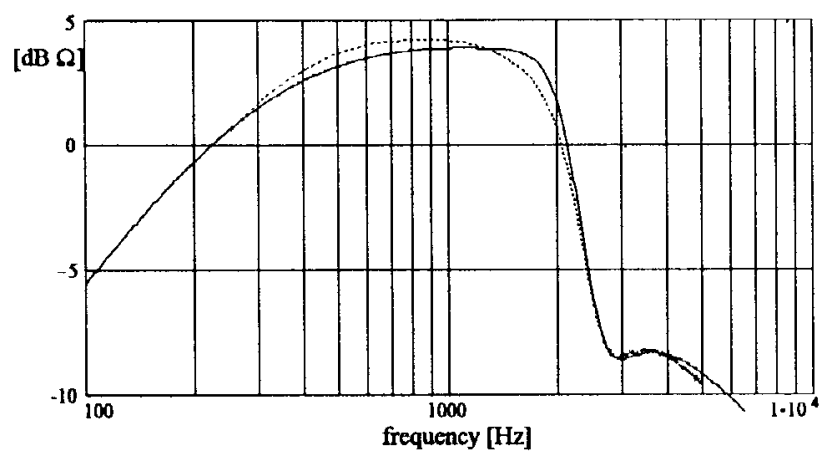

(a)

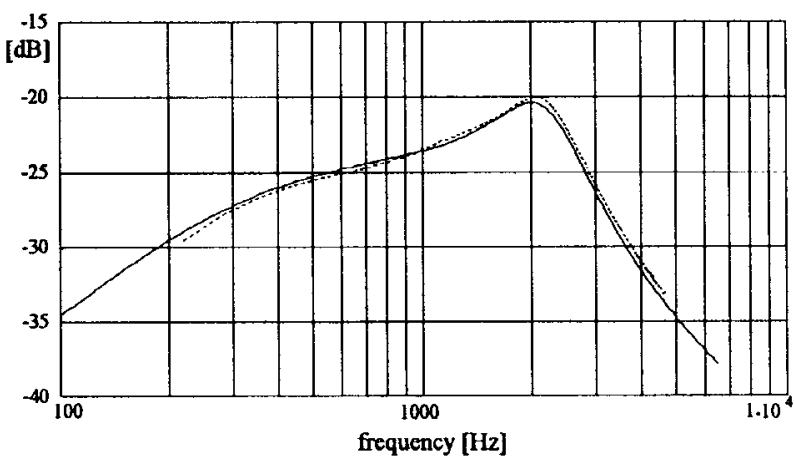

(b)

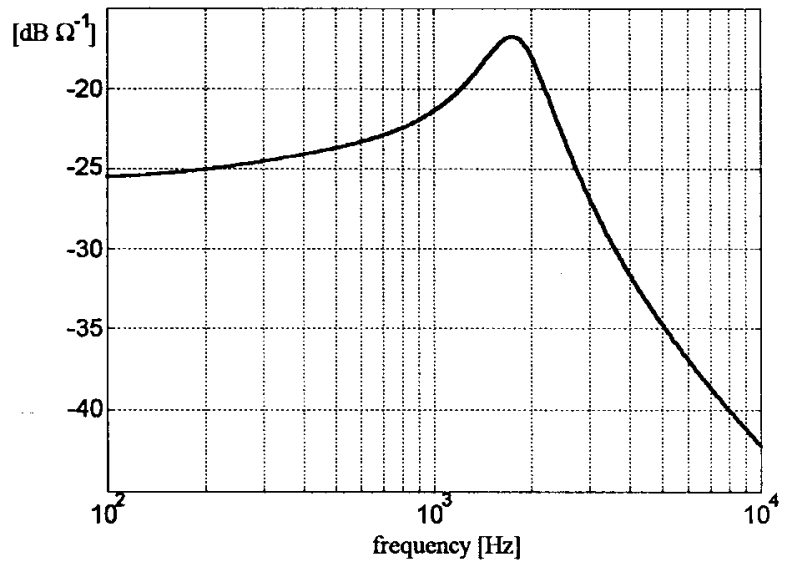

(c)

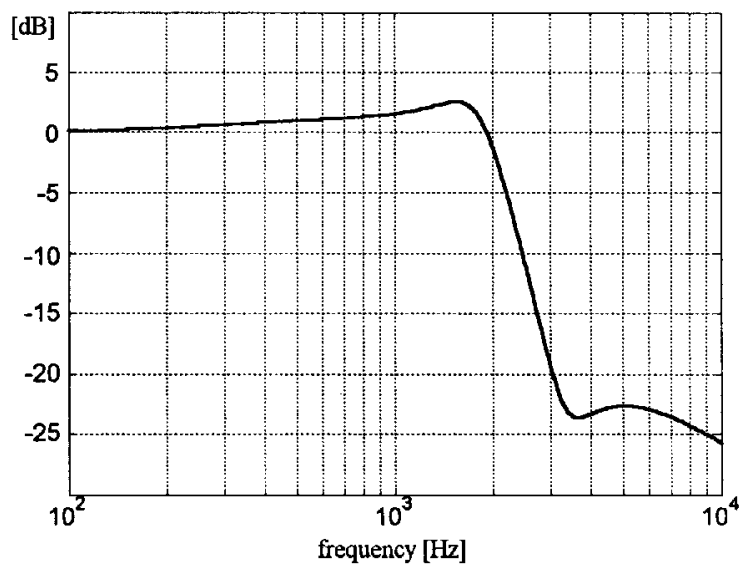

(d)

Fig. 3. (a) Output impedance, (b) audiosusceptibility (continuous line-model simulation and dotted line-circuit simulation), (c) input admittance, and (d) reference to output transfer function. 


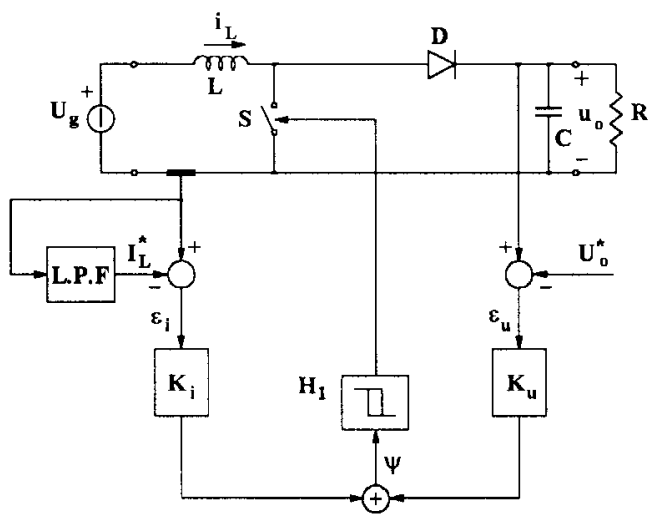

Fig. 4. Boost converter with sliding mode control.

Although a full-order control generally results in better performances, this is not true in general. For example, the audiosusceptibility of the converter in Fig. 2 is considerably better in terms of peak value and low-frequency behavior.

\section{EFFects of Control Parameter VARiations}

An important advantage of the derived small-signal model is the possibility to analyze the dynamic of the controlled system as a function of all control parameters, i.e., filter time constants and sliding coefficients $k_{i}$. This makes the design process straightforward unlike previous analyses which disregard filter time constants and do not give information on the dependence of the system dynamic on sliding coefficients.

Let us consider, for example, the Boost converter with sliding mode control shown in Fig. 4. The order of the overall system is two because we have two state variables $(N=2)$ and one low-pass filter $(M=1)$. Following the procedure given in Appendix I, we compute matrix $A_{T}$ which is given by

$$
A_{T}=\left[\begin{array}{cc}
-\frac{2}{R C}+g \frac{\delta^{\prime}\left(1-\frac{L_{\mathrm{eq}}}{R \tau}\right)}{1-g \frac{L_{\mathrm{eq}}}{R C} \delta^{\prime}} & \frac{\frac{\delta^{\prime}}{C}}{1-g \frac{L_{\mathrm{eq}}}{R C} \delta^{\prime}} \\
-\frac{g}{\tau} & 0
\end{array}\right]
$$

where $K^{T}=\left[k_{i} k_{u}\right], g=k_{u} / k_{i}, \delta^{\prime}=1-\delta, \tau$ is the filter time constant, and $L_{\mathrm{eq}}=L / \delta^{\prime 2}$. Note that coefficients $k_{i}$ and $k_{u}$ were chosen in order to make the value of $\delta$ adimensional so that $g$ becomes a conductance. The characteristic polynomial of matrix $A_{T}$ is given by the determinant of matrix $\left(s I-A_{T}\right)$

$$
\begin{aligned}
\Delta= & s^{2}+s \cdot\left[\frac{\frac{2}{R C}+g \cdot \frac{\delta^{\prime}}{C}\left(1-\frac{L_{\mathrm{eq}}}{R \tau}\right)}{1-g \frac{\delta^{\prime}}{C} \frac{L_{\mathrm{eq}}}{R}}\right] \\
& +\frac{g \delta^{\prime}}{\tau C} \frac{1}{1-g \frac{\delta^{\prime}}{C} \frac{L_{\mathrm{eq}}}{R}} .
\end{aligned}
$$

From the analysis of the coefficients of polynomial $\Delta$ (see Appendix II), we recognize that if we choose

$$
0<g<g_{\text {crit }}=\frac{R C \delta^{\prime}}{L}
$$

TABLE I

VALUES OF $\tau_{\text {crit }}$ AND $g_{\text {crit }}$ FOR BASIC CONVERTER TOPOLOGIES

\begin{tabular}{|c|c|c|c|}
\cline { 2 - 4 } \multicolumn{1}{c|}{} & Buck & Boost & Buck-boost \\
\hline gcrit $^{1}$ & $\infty$ & $\frac{\mathrm{RC} \delta^{\prime}}{\mathrm{L}}$ & $\frac{\mathrm{RC} \delta^{\prime}}{\mathrm{L} \delta}$ \\
\hline$\tau_{\text {crit }}$ & 0 & $\frac{\mathrm{L}_{\mathrm{eq}}}{\mathrm{R}+2 \frac{\mathrm{L}_{\mathrm{eq}}}{\mathrm{RC}}}$ & $\frac{\mathrm{L}_{\mathrm{cq}}}{\delta}+\left(2-\delta^{\prime}\right) \frac{\mathrm{L}_{c \mathrm{q}}}{\mathrm{RC}}$ \\
\hline
\end{tabular}

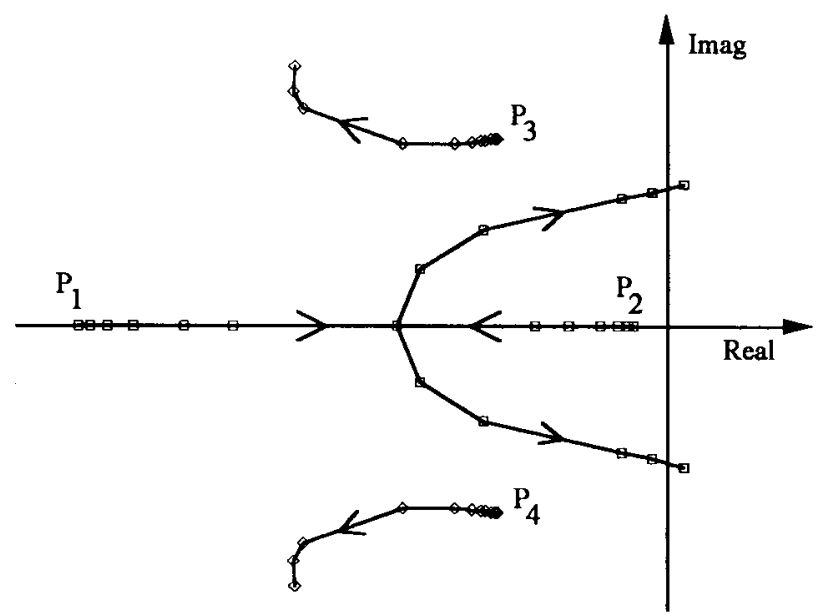

Fig. 5. Root locus of closed-loop system for variation of low-pass filter time constant of the Sepic converter in Fig. 2.

the system stability is ensured provided that

$$
\tau>\tau_{\text {crit }}=\frac{L_{\mathrm{eq}}}{R+2 \frac{L_{\mathrm{eq}}}{R C}} .
$$

It can be demonstrated that condition (14), derived under small-signal time-averaging approximation, is the same as the existence condition of the sliding mode for a Boost converter in the steady state. This is easily derived from a converter trajectory analysis (see Appendix III). The same result can be found for other basic converter topologies for which values of $\tau_{\text {crit }}$ and $g_{\text {crit }}$ are reported in Table I.

For higher order systems, for which it is not easy to derive simple conditions as (14) and (15), (11) can be used directly to observe the effects of the controller parameters on the system dynamic. For example, Fig. 5 shows the root locus resulting from different values of the filter time constant $(0.06$ $\mathrm{ms}<\tau<2 \mathrm{~ms}$ ) of the Sepic converter in Fig. 2. Too low values cause real poles $P_{1}$ and $P_{2}$ to become complex or even to cross the imaginary axis.

\section{EXPERIMENTAL RESULTS}

A Boost converter prototype, according to the scheme of Fig. 4, was used to test the validity of the above theoretical results. Converter parameters are listed in Table II.

Converter audiosusceptibility and output impedance were measured and compared with those predicted by the model. For 
TABLE II

ExPerimental Prototype Parameters

\begin{tabular}{|c|c|c|c|}
\hline $\mathrm{U}_{\mathrm{g}}=24 \mathrm{~V}$ & $\mathrm{U}_{0}=48 \mathrm{~V}$ & $\mathrm{P}_{0}=50 \mathrm{~W}$ & $\mathrm{f}_{\mathrm{S}}=50 \mathrm{kHz}$ \\
\hline $\mathrm{L}=570 \mu \mathrm{H}$ & $\mathrm{C}=22 \mu \mathrm{F}$ & $\tau=0.4 \mathrm{~ms}$ & $\mathrm{~g}=0.35$ \\
\hline
\end{tabular}

[dB $] 40$

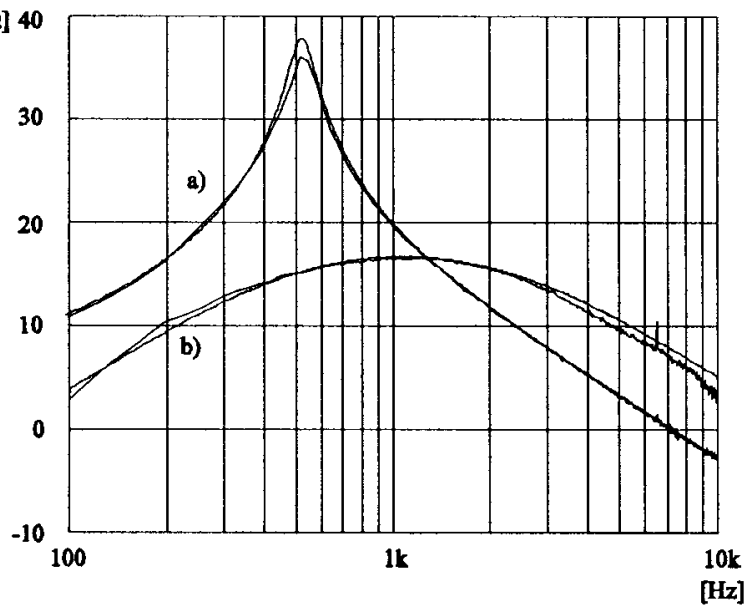

Fig. 6. Comparison between model forecast and experimental results: output impedance a) open loop and b) closed loop.

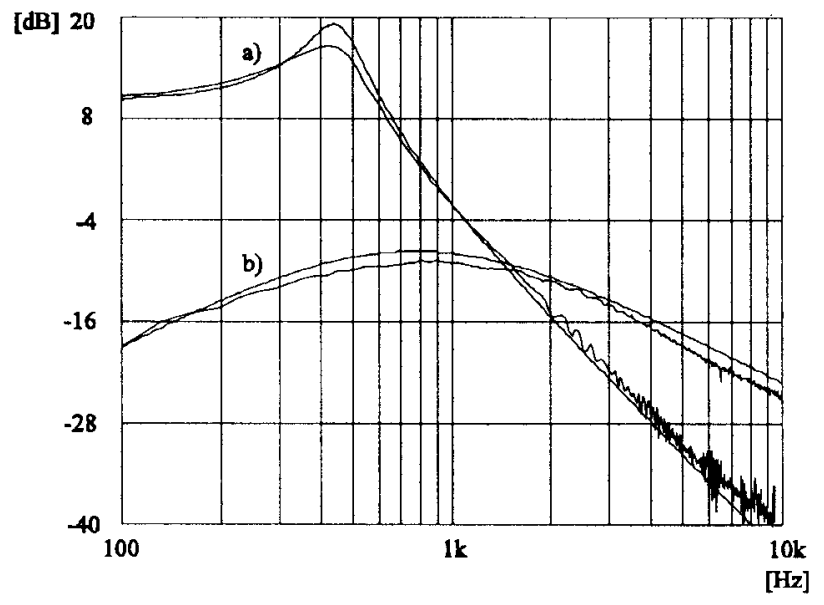

Fig. 7. Comparison between model forecast and experimental results: audiosusceptibility a) open loop and b) closed loop.

this purpose, the parameters used in the model were measured directly on the prototype, and the inductor series resistance was taken into account.

In Fig. 6, the open- and closed-loop output impedance is reported. As we can see, theoretical and experimental results agree pretty well. The sliding mode control reduces the peak in the output impedance diagram by almost $20 \mathrm{~dB}$. Fig. 7 shows the audiosusceptibility behavior in the same conditions. Once again, simulated and experimental curves look very similar, while the improvement in the audiosusceptibility peak is about $30 \mathrm{~dB}$.

Note that control causes a worsening of the dynamic characteristics at high frequencies as compared to the open-loop case. This behavior agrees with the multiloop nature of sliding mode control as explained in [7].

\section{CONCLUSION}

A small-signal model of dc-dc converters with sliding mode control is derived. It allows evaluation of closed-loop performances like audiosusceptibility, output and input impedances and reference to output transfer function.

With this model, stability as well as effects of controller parameter variations can be deeply investigated, and control parameters can be carefully selected. Simulated results of a Sepic converter and experimental results of a Boost converter are reported, showing the validity of the approach.

\section{APPENDIX I}

In order to derive the expressions of matrices in (9)-(11), it is convenient to split the vector of state variable references between internal and external variables as follows:

$$
x^{*}=\left[\begin{array}{l}
x_{\mathrm{int}}^{*} \\
x_{\mathrm{ext}}^{*}
\end{array}\right] .
$$

From (1), it follows that the derivative of sliding function $\psi$ is also zero. Thus, considering perturbed signals, we can write

$$
\dot{\hat{\psi}}=K^{T}\left(\dot{\hat{x}}-\dot{\hat{x}}^{*}\right)=K^{T} \dot{\hat{x}}-K_{\mathrm{int}}^{T} \dot{\hat{x}}_{\mathrm{int}}^{*}-K_{\mathrm{ext}}^{T} \dot{\hat{x}}_{\mathrm{ext}}^{*}=0
$$

in which the vector of sliding coefficients $K$ is divided in two terms $K_{\text {int }}$ and $K_{\text {ext }}$.

The dynamic description of the internal references is given by (7) which can be rewritten as

$$
\dot{\hat{x}}_{\mathrm{int}}^{*}=T_{1} \hat{x}_{\mathrm{int}}^{*}-T_{2} \hat{x}
$$

where $T_{1}$ is a diagonal $M \times M$ matrix, while $T_{2}$ is a $M \times N$ matrix given by

$$
T_{2}=\left[\begin{array}{ll}
T_{1} & 0
\end{array}\right]
$$

which holds in the hypothesis that the state variables in vector $x$ are ordered as in vector $x^{*}$.

Substituting (A1.3) and (5) into (A1.2) and calculating the duty cycle, we obtain (9) whose matrices are given by

$$
\begin{aligned}
& D_{1}=\left[\begin{array}{ll}
D_{11} & D_{22}
\end{array}\right] \\
& D_{2}=-\left(K^{T} C\right)^{-1} \cdot K^{T} B \\
& D_{3}=\left(K^{T} C\right)^{-1} \cdot K_{\text {ext }}^{T}
\end{aligned}
$$

where

$$
\begin{aligned}
& D_{11}=-\left(K^{T} C\right)^{-1} \cdot\left(K^{T} A+K_{\mathrm{int}}^{T} T_{2}\right) \\
& D_{22}=\left(K^{T} C\right)^{-1} \cdot K_{\mathrm{int}}^{T} T_{1} .
\end{aligned}
$$

Note that term $K^{T} C$ is scalar.

The complete description of the system is given by (10) which is obtained by substituting (9) in (5) and using (A1.3) and (8). Matrices $A_{c}, B_{c}$, and $D_{c}$ result

$$
\begin{aligned}
A_{c} & =\left[\begin{array}{cc}
A+C D_{11} & C D_{22} \\
-T_{2} & T_{1}
\end{array}\right] \\
B_{c} & =\left[\begin{array}{c}
B+C D_{2} \\
0
\end{array}\right] \\
D_{c} & =\left[\begin{array}{c}
C D_{3} \\
0
\end{array}\right] .
\end{aligned}
$$


TABLE III

\begin{tabular}{|c|c|c|c|c|}
\hline$U_{g}=15 \mathrm{~V}$ & $L_{1}=700 \mu \mathrm{H}$ & $L_{2}=360 \mu \mathrm{H}$ & $C_{1}=6.8 \mu \mathrm{F}$ & $C_{2}=250 \mu \mathrm{F}$ \\
\hline$n=1.5$ & $U_{2}=20 \mathrm{~V}$ & $R=20-200 \Omega$ & $g=1$ & $\tau=0.6 \mathrm{~ms}$ \\
\hline
\end{tabular}

Lastly, in order to derive the matrices of the reduced order system (11), let us consider nonzero the first element $k_{1}$ of vector $K$. Thus, from (1) and (A1.1) we can write

$K^{T}\left(\hat{x}-\hat{x}^{*}\right)=\left[\begin{array}{ll}K^{T} & -K_{\mathrm{int}}^{T}\end{array}\right] \cdot \hat{x}^{\prime}-K_{\mathrm{ext}}^{T} \cdot \hat{x}_{\mathrm{ext}}^{*}=0 . \quad$ (A1.8)

Solving for the first state variable and substituting in (10), we obtain (11) in which we have

$$
\begin{aligned}
\hat{x}^{\prime \prime} & =\hat{x}^{\prime}(2: N+M) \\
A_{T} & =A_{c}(2: N+M, 2: N+M)+A_{c}(2: N+M, 1) \cdot F_{1} \\
B_{T} & =B_{c}(2: N+M,:) \\
D_{T 1} & =A_{c}(2: N+M, 1) \cdot F_{2} \\
D_{T 2} & =D_{c}(2: N+M,:)
\end{aligned}
$$

where

$$
\begin{aligned}
& F_{1}=-\frac{1}{k_{1}} \cdot\left[K^{T}(2: N)-K_{\mathrm{int}}^{T}\right] \\
& F_{2}=\frac{1}{k_{1}} \cdot K_{\mathrm{ext}}^{T}
\end{aligned}
$$

and the notation $A(n: m, j: k)$ means the submatrix obtained from matrix $A$ taking rows from index $n-m$ and columns from index $j-k$, while the symbol : alone means all.

For example, for a Boost converter the previous matrices are given by the following expressions:

$$
\begin{aligned}
A & =\left[\begin{array}{ll}
0 & -\frac{\delta^{\prime}}{L} \\
\frac{\delta^{\prime}}{C} & -\frac{1}{R C}
\end{array}\right] \quad B=\left[\begin{array}{l}
\frac{1}{L} \\
0
\end{array}\right] \quad C=\left[\begin{array}{c}
\frac{U_{g}}{\delta^{\prime} L} \\
-\frac{U_{g}}{\delta^{\prime 2} R C}
\end{array}\right] \\
T_{1} & =\left[\begin{array}{cc}
-\frac{1}{\tau}
\end{array}\right] \quad T_{2}=\left[\begin{array}{ll}
-\frac{1}{\tau} & 0
\end{array}\right] \\
A_{c} & =\left[\begin{array}{ccc}
\frac{\eta}{\tau}\left(1-\frac{\delta^{\prime} \tau g}{C}\right) & \frac{2 \eta g}{R C} & -\frac{\eta}{\tau} \\
\frac{\eta \delta^{\prime}}{C}\left(1-\frac{L_{\mathrm{eq}}}{\tau R}\right) & -\frac{2 \eta}{R C} & \frac{\eta \delta^{\prime} L_{\mathrm{eq}}}{\tau R C} \\
\frac{1}{\tau} & 0 & -\frac{1}{\tau}
\end{array}\right] \\
B_{c} & =\frac{\eta}{\delta^{\prime} R C}\left[\begin{array}{c}
-g \\
1 \\
0
\end{array}\right] \quad D_{c}=\eta g\left[\begin{array}{c}
1 \\
\delta^{\prime} \frac{L_{\mathrm{eq}}}{R C} \\
0
\end{array}\right] \\
B_{T} & =\frac{\eta}{\delta^{\prime} R C}\left[\begin{array}{l}
1 \\
0
\end{array}\right] \quad D_{T 1}=\left[\begin{array}{c}
\frac{\eta \delta^{\prime} g}{C}\left(1-\frac{L_{\mathrm{eq}}}{\tau R}\right) \\
\frac{g}{\tau}
\end{array}\right] \\
D_{T 2} & =g \eta\left[\begin{array}{c}
\delta^{\prime} \frac{L_{\mathrm{eq}}}{R C} \\
0
\end{array}\right]
\end{aligned}
$$

where

$$
\eta=\frac{1}{1-g \frac{L_{\mathrm{eq}}}{R C} \delta^{\prime}}
$$

and $A_{T}$ is given by (12).

\section{APPENDIX II}

In order to derive conditions (14) and (15), which ensure system stability for a Boost converter with sliding mode control, we analyze the sign of coefficients of the characteristic polynomial $\Delta$ (13). Calling $r_{1}$ and $r_{2}$ the roots of $\Delta$, we can write

$$
\Delta=s^{2}-s \cdot\left(r_{1}+r_{2}\right)+r_{1} \cdot r_{2} .
$$

The system is stable if both $r_{1}$ and $r_{2}$ have a negative real part, i.e., if

$$
r_{1} \cdot r_{2}>0
$$

and

$$
r_{1}+r_{2}<0 \text {. }
$$

From (13), (A2.2), and (A2.3) the following inequalities result:

$$
\begin{array}{r}
\frac{g}{\tau} \frac{\delta^{\prime}}{C} \frac{1}{1-g \frac{\delta^{\prime}}{C} \frac{L_{\mathrm{eq}}}{R}}>0 \\
\frac{\frac{2}{R C}+g \frac{\delta^{\prime}}{C}\left(1-\frac{L_{\mathrm{eq}}}{R \tau}\right)}{1-g \frac{\delta^{\prime}}{C} \frac{L_{\mathrm{eq}}}{R}}>0 .
\end{array}
$$

From (A2.4) it results

$$
0<g<g_{\text {crit }}=\frac{R C}{\delta^{\prime} L_{\mathrm{eq}}}=\frac{R C \delta^{\prime}}{L}
$$

which is condition (14). From (A2.5), taking into account (A2.6), it results

$$
\tau>\frac{L_{\mathrm{eq}}}{R} \frac{1}{1+\frac{2}{R \delta^{\prime} g}}
$$

where, substituting the maximum value $g_{\text {crit }}$ for $g$ given by (A2.6), the minimum value $\tau_{\text {crit }}$ as given by (15) results.

\section{APPENDIX III}

As already mentioned, the existence condition requires that the phase trajectories are directed toward the sliding surface in a small volume around the surface itself. This statement translates into conditions [1], [2]

$$
\begin{aligned}
& \lim _{\psi \rightarrow 0^{+}} \frac{d \psi}{d t}<0 \\
& \lim _{\psi \rightarrow 0^{-}} \frac{d \psi}{d t}>0 .
\end{aligned}
$$

Now, in order to satisfy existence conditions [1], [4], we choose the control strategy that closes the switch when the sliding function $\psi$ is negative and opens the switch when $\psi$ 
is positive. Thus, writing the expression of $\dot{\psi}=K^{T} \dot{x}$ for the Boost converter in the two situations corresponding to the different values of the switch status (open for the first one and closed for the second one), the above inequalities (A.3.1) give

$$
\begin{aligned}
& \frac{k_{u}}{k_{i}}<\frac{R C}{L} \cdot \frac{U_{g}}{u_{C}} \\
& \frac{k_{u}}{k_{i}}<\frac{R C}{L} \cdot \frac{u_{C}-U_{g}}{R i_{L}-u_{C}}
\end{aligned}
$$

where $u_{C}$ is the instantaneous voltage of the output filter capacitor, and $i_{L}$ is the input current. Now, if we consider a small volume around the operating point in the phase plane (small-signal approximation), we can use the nominal capacitor voltage in (A3.2a) and (A3.2b) which is equal to the output voltage. With this approximation, simple calculations show that (A.3.2b) is equivalent to (A.3.2a), thus obtaining condition (14).

\section{APPENDIX IV}

The parameter values of the Sepic converter of Fig. 2 are given in Table III on the previous page.

\section{REFERENCES}

[1] V. I. Utkin, Sliding Mode and Their Application in Variable Structure System. Moscow: MIR, 1974.

[2] U. Itkis, Control Systems of Variable Structure. New York: Wiley, 1976.
[3] R. Venkataramanan, A. Sabanovic, and S. Cuk, "Sliding-mode control of dc-to-dc converters," IECON, 1985.

[4] P. Mattavelli, L. Rossetto, G. Spiazzi, and P. Tenti, "General-purpose sliding-mode controller for $\mathrm{dc}-\mathrm{dc}$ converter applications," in PESC Conf. Proc., 1993, pp. 609-615.

[5] R. D. Middlebrook and S. Cuk, Advances in Switched-Mode Power Conversion. Pasadena: Teslaco, 1981, pp. 73-89.

[6] P. Mattavelli, L. Rossetto, G. Spiazzi, and P. Tenti, "Sliding mode control of SEPIC converters," in Proc. European Space Power Conf. (ESPC), Graz, Austria, 1993, pp. 173-178.

[7] R. B. Redley, B. H. Cho, and F. C. Lee, "Analysis and interpretation of loop gains of multiloop-controlled switching regulators," IEEE Trans. Power Electron., vol. 3, no. 4, pp. 489-498, Oct. 1988.

Paolo Mattavelli (S'92-M'96), for a photograph and a biography, see this issue, p. 86.

Leopoldo Rossetto (M'95), for a photograph and a biography, see this issue, p. 86.

Giorgio Spiazzi (S'92-M'94), for a photograph and a biography, see this issue, p. 86. 